\title{
Highly Homogeneous Carbon Nanotube-Polycaprolactone Composites with Various and Controllable Concentrations of Ionically-Modified-MWCNTs
}

\author{
Hae-Hyoung Lee, ${ }^{\dagger, \S}$ Ueon Sang Shin, ${ }^{\ddagger, \$, *}$ Guang-Zhen Jin, ${ }^{\S}$ and Hae-Won Kim ${ }^{\dagger, \ldots, \&, *}$ \\ †Department of Biomaterials Science, School of Dentistry, Dankook University, Korea. ${ }^{*}$ E-mail: kimhw@dku.edu \\ ${ }^{\star}$ Biomaterials and Tissue Engineering Lab., Department of Nanobiomedical Science \& WCU Research Center, \\ Dankook University, Cheonan 330-714, Korea. E-mail: usshin12@dankook.ac.kr \\ ${ }^{\S}$ Institute of Tissue Regeneration Engineering (ITREN), Dankook University, Cheonan 330-714, South Korea \\ Received June 18, 2010, Accepted November 3, 2010
}

\begin{abstract}
For the fabrication of multifunctional biopolymer nanocomposites in the combination of carbon nanotubes (CNTs), recently increasing attention has been paid to an effective homogenization of CNTs within polymer matrices and a fine tuning of the concentration. We developed an efficient method to produce homogeneous CNT-polycaprolactone nanocomposites with various and controllable CNT concentrations using an ionically-modified multi-walled CNT, MWCNT-Cl. The modified MWCNTs could be homogeneously dispersed in tetrahydrofuran (THF). Polycaprolactone (PCL) as a biodegradable and biocompatible polymer was smoothly dissolved in the homogeneous MWCNT-Cl/ THF solution without agglomeration of MWCNT-Cl. The physicochemical and mechanical properties of the resultant nanocomposites were examined and the biological usefulness was briefly assessed.
\end{abstract}

Key Words: Multi-walled carbon nanotubes (MWCNTs), Polymer nanocomposite, Polycaprolactone (PCL)

\section{Introduction}

Carbon nanotubes (CNTs) have been widely recognized as new lightweight nanofillers with excellent electrical ${ }^{1}$ and mechanical $^{2}$ properties. Accordingly, many researchers are interested in their use for the fabrication of multifunctional polymer nanocomposites with improved properties even in the presence of small amounts of CNTs. Despite the positive prospect of CNTs as a class of future materials, their use has been restricted due to difficulties in their homogenization within most polymer matrices and the weak interfacial interaction between CNT and polymer matrices. Many attempts have been made to mechanically mix the pristine CNTs within organic solutions of polymers or directly in polymer melts, which however resulted in significant level of CNT-lump presence in the matrices. ${ }^{3}$

Recent focus has been put on the functionalization of CNTs to enable their homogeneous dispersion in solvents and to improve their interfacial interaction with polymer-matrices. Among the functionalization methods, oxidative cutting of CNTs using a strong oxidant in an acidic medium at high temperature has been a case of in point. ${ }^{4,5}$ However, the harsh reaction conditions cause significant damage to the nanotubes, resulting in shortened nanotubes that ultimately lose their primary properties and even become sensitive to air. The resultant composites showed limited improvement in mechanical and electrical properties due to the CNTs degradation and still not enough solubility in solvent. ${ }^{6}$ A chemical tethering of the matrix molecules to the carboxylic functional groups has also been extensively studied to enhance the interfacial interactions between CNTs and polymer matrix. The clear advantage for this approach may be that CNT aggregation in the composites can be greatly prevented, leading to mechanical improvement. ${ }^{7-12}$ However, the manufacturing process was time-consuming and the electrical conductivity could not be much enhanced. This was believed that the additional anchoring of polymer molecules on the CNT surface caused a kind of blocking or encapsulation of every nanotube molecule, thereby leading to hindrance of the interconnection of the CNTs. For these reasons, it is highly desirable to develop an efficient and simple method to control concentration of CNTs, as well as individually to dissolve CNTs in polymer matrices, resulting in increment of the nanotube-interconnectivity, which translates the individual nanotube properties into the whole composite.

Polycaprolactone (PCL) is one of a few degradable biopolymers with FDA-approval, and thus has long been used as medical devices, including drug carriers and tissue engineering scaffolds. $^{13,14}$

The goal in the manuscript is the development of an efficient method to produce homogeneous CNT-biopolymer nanocomposites with various and controllable CNT concentrations. For this, we prepared chemically-modified MWCNT derivatives, MWCNT-X $\left(\mathrm{X}=\mathrm{SbF}_{6}, \mathrm{Cl}\right.$, etc. $)$ and homogeneous MWCNT$\mathrm{Cl}$ solution and MWCNT-Cl/PCL composite solution in tetrahydrofurane (THF). The approach to using the ionically-modified CNTs is considered to open a new door to the class of CNT-added polymer nanocomposites, including those for biomedical applications.

\section{Experimental Part}

Materials. Pristine multi-walled carbon nanotubes ( $p$ MWC NTs) synthesized by chemical vapor deposition (CVD) were purchased from ILJIN Nanotech Co., Ltd. (Seoul, Korea). Ionic liquids, [bmim]X (X $=\mathrm{SbF}_{6}$, OTf, $\left.\mathrm{Cl}\right)$, were purchased from C-Tri Co., Ltd., Korea, and used without further purification. Their water contents were $<50 \mathrm{ppm}$ (determined by Karl-Fisher titration). According to the procedure reported previously, ${ }^{15}$ the Lewis acidic ionic liquid, [bmim] $\left[\mathrm{Sb}_{2} \mathrm{~F}_{11}\right]$, was prepared by 
mixing [bmim] $\left[\mathrm{SbF}_{6}\right]$ and $\mathrm{SbF}_{5}$ in same equivalent under an Ar atmosphere. $\mathrm{SbF}_{5}$ was transferred to the dried [bmim] $\left[\mathrm{SbF}_{6}\right]$ under Ar atmosphere over $10 \mathrm{~min}$ to afford [bmim] $\left[\mathrm{Sb}_{2} \mathrm{~F}_{11}\right]$. Organic solvents were dried and distilled under an Ar atmosphere prior to use. Polycaprolactone (PCL) with Mn of $8 \times 10^{4}$ $\mathrm{g} / \mathrm{mol}$ and tetrahydrofuran (THF) were purchased from Aldrich and used without purification.

Preparation of PCL/MWCNT-Cl Composites (PCLNTs). Under an Ar atmosphere, [bmim] $\left[\mathrm{Sb}_{2} \mathrm{~F}_{11}\right](0.16 \mathrm{~g}, 0.28 \mathrm{mmol})$ as a Lewis acidic IL was mixed well with a suspension of $p$ MWCNTs (200 mg, 2.8 mmol based on $\mathrm{C}_{6}$ unit of nanotubes) in $10 \mathrm{~mL}$ of methylene chloride. $200 \mathrm{mg}$ (2.8 mmol) of THF was slowly added to the suspension, followed by sonication for 15 min. After quenching with $0.1 \mathrm{~mL}$ water, the resulting product, MWCNT-SbF 6 , was filtered through a Teflon membrane and washed with acetone and methylene chloride many times. After drying in a vacuum, MWCNT-SbF 6 was obtained in yields of $367 \mathrm{mg}$ as an air-stable black powder. MWCNT-SbF 6 (300 $\mathrm{mg} ; 108 \mathrm{mg}$ (0.5 mmol) $\left.\mathrm{SbF}_{6}{ }^{-}\right)$and $\mathrm{NaCl}(290 \mathrm{mg}, 5 \mathrm{mmol})$ were added to a mixture of water $(30 \mathrm{~mL})$ and methanol $(30 \mathrm{~mL})$. The mixture was sonicated for $5 \mathrm{~min}$ and additionally stirred for $3 \mathrm{~h}$ at $25^{\circ} \mathrm{C}$. The resulting MWCNT-Cl powder was filtered through a Teflon membrane and washed with water, acetone, and methylene chloride in that order. After drying in a vacuum, the product, MWCNT-Cl, was obtained in a yield of $126 \mathrm{mg}$ as an air-stable black powder.

A homogeneous solution of MWCNT-Cl was prepared by dissolving MWCNT-Cl (50 mg) in $100 \mathrm{~mL}$ THF under ultrasonic vibration for $1 \mathrm{~min}$. Subsequently, $200 \mathrm{mg}$ of PCL were

Table 1. Material amounts used for preparing PCLNT composites and sample designations

\begin{tabular}{ccccc}
\hline Entry & $\begin{array}{c}\text { PCL } \\
(\mathrm{mg})\end{array}$ & $\begin{array}{c}\text { MWCNT-Cl/THF } \\
\text { (mL) }\end{array}$ & $\begin{array}{c}\text { MWCNT-Cl } \\
(\text { wt } \%)\end{array}$ & $\begin{array}{c}\text { Composite } \\
\text { Name }\end{array}$ \\
\hline 1 & 200 & 0 & 0 & PCL \\
2 & 200 & 1 & 0.25 & PCLNT-1 \\
3 & 200 & 2 & 0.5 & PCLNT-2 \\
4 & 200 & 3 & 0.75 & PCLNT-3 \\
5 & 200 & 4 & 1.0 & PCLNT-4 \\
\hline
\end{tabular}

${ }^{a}$ MWCNT-Cl/THF solution has a concentration of $0.5 \mathrm{mg} / \mathrm{mL}$. dissolved in various amounts of the MWCNT-Cl/THF solution ( $1 \sim 4 \mathrm{~mL}$ ), resulting in the formation of highly clean and homogeneous PCL-MWCNT-Cl (PCLNT) solutions (Table 1 and Figure 1). The composites (PCLNT-1 4) solidified after evaporating the solvent were brought to a hot presser to produce $0.01 \mathrm{~mm}$-thick films (at $50^{\circ} \mathrm{C}$ ). The resultant composites containing various MWCNT-Cl concentrations are designated as shown in Table 1.

Instruments and Characterization. FT-Raman spectra of the composite samples were recorded with a Raman spectrometer (Bruker, FRA 106/S) using an Nd-YAG-laser (1064 nm excitation wavelength). The samples were analyzed in dry condition. UV-vis-NIR absorption measurements were taken using a Cary 5000 spectrophotometer (Varian, CA). The optical characteristics of the composites were monitored based on their absorbance at wavelengths ranging from 190 to $1400 \mathrm{~nm}$. All measurements were made at room temperature, and all spectra were corrected by a background offset. The thermogravimetric analysis (TGA) was conducted using Seiko Exstar 6000 (TG/ DTA6100) (SEICO INST., JAPAN) with a heating rate of 10 ${ }^{\circ} \mathrm{C} / \mathrm{min}$ at temperatures ranging from 20 to $600{ }^{\circ} \mathrm{C}$ under air. Differential scanning calorimetry (DSC 60) was carried out with a heating rate of $20{ }^{\circ} \mathrm{C} / \mathrm{min}$ at temperatures ranging from 20 to $100{ }^{\circ} \mathrm{C}$. Scanning electron microscopic images (SEM) were obtained using a JSM7000F (JEOL, Tokyo, Japan) and HITACHI S-3000H (Japan) microscopes.

The tensile tests were performed using an Instron universal material testing system (Instron 3344) at room temperature. Specimens with a dimension of $10 \mathrm{~mm} \times 3 \mathrm{~mm} \times 0.01 \mathrm{~mm}$ were drawn at a speed of $5 \mathrm{~mm} / \mathrm{min}$ and the average value of four specimens was reported here. Homogeneity and dispersion stability analysis for the PCLNT-4 solution were performed by using TurbiScane LAb (Leanontech, a pulsed near infrared light source $(880 \mathrm{~nm})$. The composite solution of $35 \mathrm{~mm}$ height was lengthwise scanned every minute for 30 minutes at $30^{\circ} \mathrm{C}$. The light backscattered by the sample solution $\left(135^{\circ}\right)$ was measured. The elemental analysis was carried out by elemental analyzer using a FLASH EA 1112 (Thermo Elemental, USA) with an error range of $0.1 \%$. The samples were enough dried under vacuum before the measurement. The quantitative analysis for antimony ( $\mathrm{Sb}$ ) was performed by ICP-OES using an Iris Intrepid ICP-OES (Thermo Elemental, USA). (a)

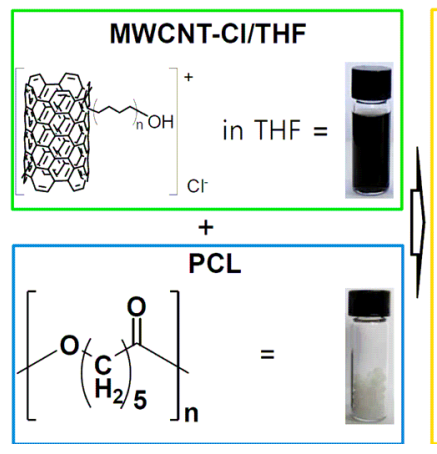

(b)

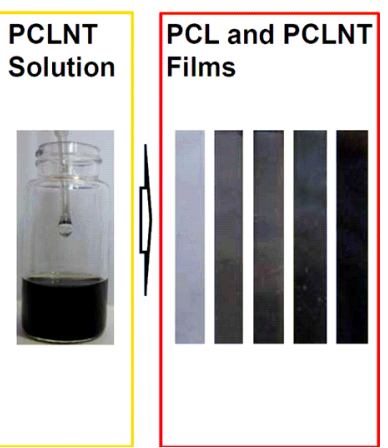

Figure 1. Schematic diagram for the modification of $p$ MWCNTs and photographs of (a) MWCNT-Cl solution in THF and PCL in granular type, (b) PCLNT-4 solution in THF, and (c) neat PCL and PCLNT-1 $\sim 4$ films (from left to right). 
Cell Viability Test. Murine-derived preosteoblast (MC3T3E1) was used to evaluate the cell response to the PCLNT nanocomposites. Pure PCL film was used for comparison. The samples were cut to a disc with a diameter of $6 \mathrm{~mm}$ and contained in each well of 96-well plates. Prior to seeding cells, samples were sterilized with $70 \%$ ethanol. Cells were seeded at $4 \times 10^{4}$ cells per sample, and then cultured for up to 7 days, under standard cell culture conditions $\left(37^{\circ} \mathrm{C}, 5 \% \mathrm{CO}_{2}\right.$ and $95 \%$ humidity) using Dulbecco's modified eagle medium supplemented with $10 \%$ fetal bovine serum and $1 \%$ penicillin/streptomycin. Cell morphology grown on the samples was examined with fluorescence microscopy after staining the nuclei of cells viable, after culturing for 3 days. The cell viability on the PCLNT nanocomposites and PCL was assessed by measuring the mitochondrial activity of viable cells using an MTS method. CellTiter 96 Aqueous One Solution (Promega) was added to the cell cultured sample and assessed colorimetrically at an absorbance of $490 \mathrm{~nm}$.

Statistical Analysis. Data were represented as mean \pm standard deviation (S.D.), and comparison between groups was carried out using student $t$-test. Statistical significance was considered at $\mathrm{p}<0.05$ and $\mathrm{p}<0.01$.

\section{Results and Discussion}

Characterization of MWCNT Derivatives and Homogeneity of MWCNT Solutions. To determine the precise amounts of every element in $p$ MWCNTs and the derivatives, quantitative elemental analyses were performed after sufficient dry under a vacuum. The analysis for carbon, hydrogen and nitrogen was carried out by means of an elemental analyzer using a FLASH EA 1112 (Thermo Elemental, USA) with an error range of $0.1 \%$. The quantitative analysis for antimony $(\mathrm{Sb})$ was performed by ICP-OES using an Iris Intrepid ICP-OES (Thermo Elemental, USA), resulting in a content of $18.6 \mathrm{mg}$ of $\mathrm{Sb}$ atom (or $36.0 \mathrm{mg}$

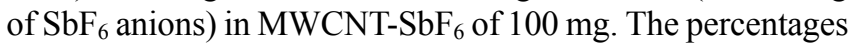
of $\mathrm{C}, \mathrm{H}, \mathrm{N}$ and $\mathrm{Sb}$ based on the weight of the sample were summarized in Table 2. At this point, one may notice that the total

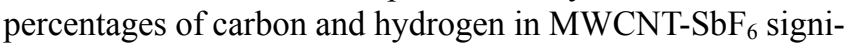
ficantly decreased from $93.9 \%$ of $p$ MWCNTs to $51.5 \%$. However, after exchanging the $\mathrm{SbF}_{6}$ anion with chloride, the percentages of both atoms in the samples again increased to 91.1 and $92.9 \mathrm{wt} \%$, respectively. It must be assumed that the remaining percentages are attributed to the presence of oxygen. Furthermore, the dissolving patterns of the two derivatives, MWCNT$\mathrm{SbF}_{6}$ and $\mathrm{Cl}$, were greatly different. In fact, the solubility of MWCNT-Cl in organic solvents (THF, $p$-xylene, MC) was re-

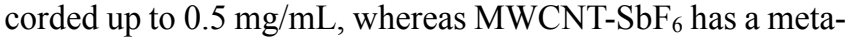
stable solubility in organic solvents.

Table 2. Elemental Analysis of $p$ MWCNTs and MWCNT-SbF 6 and $-\mathrm{Cl}$

\begin{tabular}{lcccc}
\hline \multirow{2}{*}{ MWCNTs } & \multicolumn{4}{c}{ Weight percentages (\%) } \\
\cline { 2 - 5 } & $\mathrm{C}$ & $\mathrm{H}$ & $\mathrm{N}$ & $\mathrm{Sb}$ \\
\hline pMWCNTs & 93.8 & 0.14 & 0.0 & - \\
MWCNT-SbF $_{6}$ & 50.8 & 0.75 & 0.0 & 18.6 \\
MWCNT-Cl & 90.8 & 0.33 & 0.1 & - \\
\hline
\end{tabular}

In order to produce homogeneous nanocomposite films, the dispersion stability and homogeneity of MWCNT-Cl and PC LNT solutions in THF should be verified. These were confirmed by measuring the backscattered light of a pulsed near infrared light source of $880 \mathrm{~nm}$ wavelength (Figure 2). During the scanning experiment of the most concentrated sample PCLNT 4 for $30 \mathrm{~min}$, any noticeable change of the light fluxes backscattered by the sample solution was not detected, demonstrating highly stable and uniform dispersion of nanotube molecules in the polymer solution. After standing even for several months the composite solution maintained the homogeneity as well.

Morphology and Characterization of Nanocomposites. The surface morphologies of samples were examined by SEM, as shown in Fig. 3a-c. The images showed the bulk surface-nanostructure of PCLNT-4 films, which is very similar to that of pure PCL film (Figure 3). Although the existence of carbon nanotubes inside of the composite film could be easily confirmed

(a)

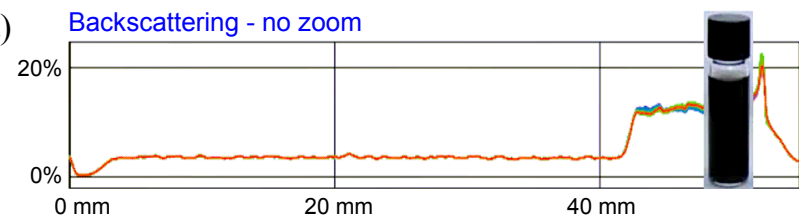

(b) Backscattering - no zoom

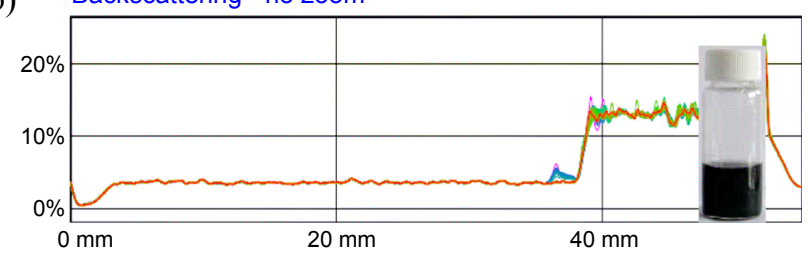

Figure 2. Homogeneity and dispersion stability of (a) MWCNT-Cl and (b) PCLNT-4 solutions in THF.
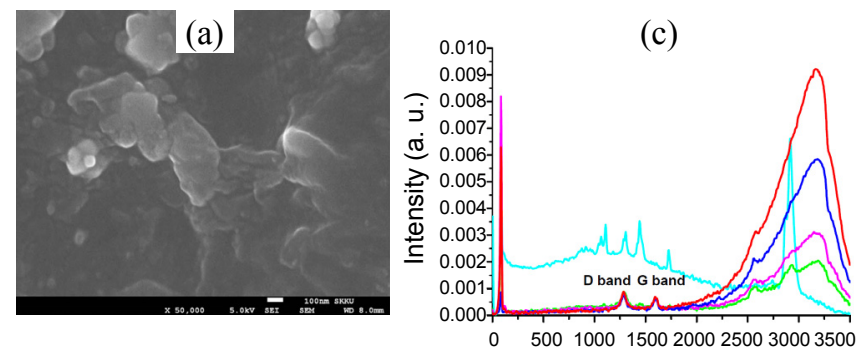

Raman shift $\left(\mathrm{cm}^{-1}\right)$
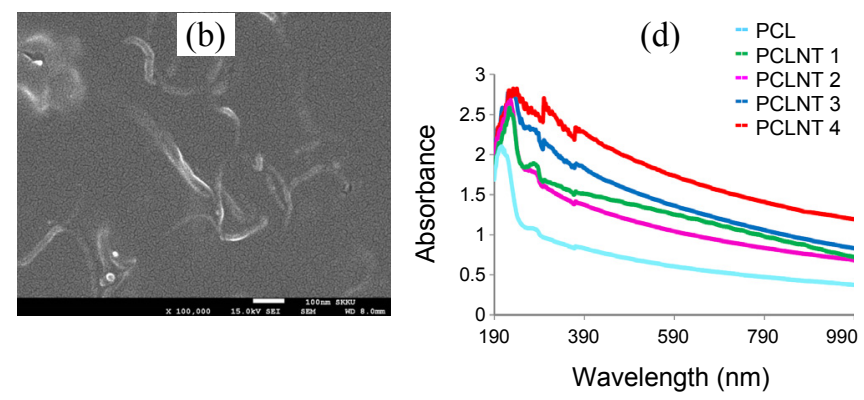

Figure 3. High resolution SEM images of PCLNT-4 film. (a) before and (b) after burning; (c) Raman and (d) UV-vis-NIR spectra of PCL and PCLNT-1 $\sim \mathbf{- 4}$. 
from the color difference, however, it was difficult to examine their dispersion status from microscopic methods including transmission electron microscopy (TEM). To confirm the presence of carbon nanotubes, a portion of the polymer matrix was burnt out at $300{ }^{\circ} \mathrm{C}$. The SEM image clearly revealed the presence of MWCNTs which were uniformly and densely dispersed in the polymer matrix (Figure 3b, including inset).

To measure the relative differences in the carbon nanotube concentrations in PCLNT-1 -4, a Raman spectrometer was employed (Figure 3c). As expected, the Raman spectra of PCLNT-1 - 4 clearly showed several characteristic peaks at ca. 1290 (D band), 1600 ( $\mathrm{G}$ band), and $3168 \mathrm{~cm}^{-1}$. The origin of the third band at $c a .3169 \mathrm{~cm}^{-1}$ which has been observed also in Raman spectrum of pristine MWCNTs is not clear. However, what is certain is that the magnitudes of the bands become larger in proportion to the increasing amount of nanotubes. These results clearly indicate that the concentration of MWCNTs in the composites is tunable simply by adjusting the used quantity of the MWCNT-Cl/THF solution. The UV-vis-NIR absorption spectra of PCL and PCLNT-1 $\sim \mathbf{- 4}$ are shown in Figure $3 \mathrm{~d}$. The spectrum of PCL exhibits a characteristic absorbance with a maximum at $212 \mathrm{~nm}$, whereas the bands with maxima in the spectra of PCLNT-1 -4 are consistently shifted up to $278 \mathrm{~nm}$ as the MWCNT-contents increase. Moreover, for the composites with higher MWCNT-contents, the characteristic absorbance is more and more slowly decreased in intensity till to reach the near-infrared region.

As shown in Figure 4a, the thermal stability of PCLNT 1-4 was investigated by thermogravimetric analysis (TGA). The decomposition curves of the neat PCL and the CNT-derivative, used as references, showed intensive weight-loss in the range of $200-400$ and $500-600{ }^{\circ} \mathrm{C}$, respectively. PCLNT 1-4 were found to be decomposed between 200 and $400{ }^{\circ} \mathrm{C}$ with slightly different patterns. In general, increased carbon nanotube content reduced the rate of decomposition as shown in the inset. The thermal stability increased even in the presence of small amount of MWCNT-Cl which suggests the homogeneous dispersion in the composites. Differential scanning calorimetry (DSC) was used to observe the melting behavior of neat PCL and its nanocomposites during heating at a rate of $10{ }^{\circ} \mathrm{C} / \mathrm{min}$ (Figure $4 \mathrm{~b}$ ). The melting temperatures (Tm) of PCLNT 1 and 2 were $60.94{ }^{\circ} \mathrm{C}$ and $60.54{ }^{\circ} \mathrm{C}$, respectively, while that of the
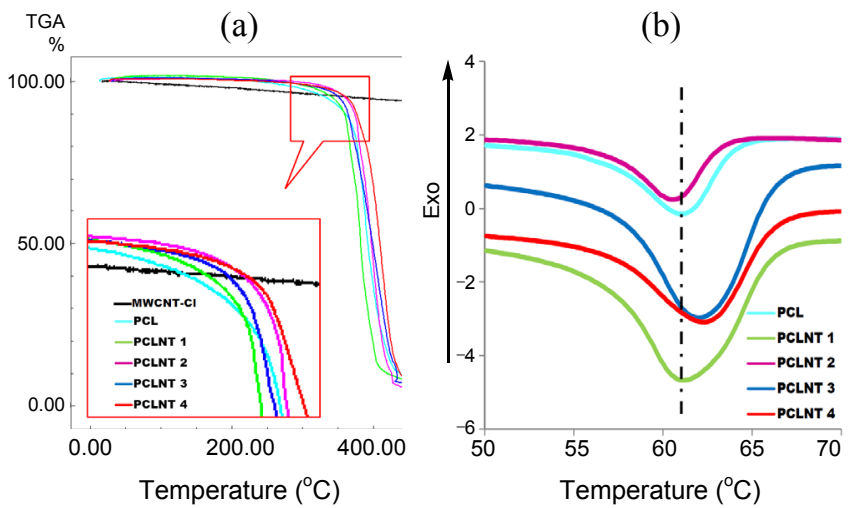

Figure 4. (a) TGA thermograms and (b) DSC curves of MWCNT-Cl, PCL, and PCLNT 1-4.
Table 3. Tensile strengths and elastic modulus of PCL and PCLNT films

\begin{tabular}{ccc}
\hline $\begin{array}{c}\text { CNT content } \\
(\text { wt \%) }\end{array}$ & $\begin{array}{c}\text { Tensile strength } \\
(\mathrm{MPa})\end{array}$ & $\begin{array}{c}\text { Elastic Modulus } \\
(\mathrm{MPa})\end{array}$ \\
\hline 0 & 17.0 & 44.7 \\
0.25 & 19.2 & 76.8 \\
0.5 & 19.1 & 72.1 \\
0.75 & 17.3 & 69.5 \\
1 & 19.8 & 75.2 \\
\hline
\end{tabular}

neat PCL was $61.07{ }^{\circ} \mathrm{C}$. Conversely, the Tm of composites with higher CNT contents (PCLNT 3 and 4) increased to 61.5 and $61.89{ }^{\circ} \mathrm{C}$, respectively.

The mechanical properties of the composites were evaluated by applying a tensile stress at room temperature. The tensile strengths of the PCL and PCLNT films are summarized in Table 3 . The presence of MWCNT-Cl generally led to increased tensile strength and elastic modulus of the samples. Indeed, even in the presence of relatively small amount of nanotubes ( $0.25-1.0 \mathrm{wt} \%)$, the strength increased by about $13 \%$. The contents at $0.25-0.5 \mathrm{wt} \%$ were proven to be the most effective in improving the mechanical strength of PCL. In terms of elastic modulus, the presence of MWCNT-Cl generally led to increased by $55.5-71.8 \%$. The clear improvements even due to such a small content of nanotubes may demonstrate the homogeneous dispersion of MW CNT-Cl within PCL matrix.

Cell Responses. To find biomedical applications of the produced PCLNT nanocomposites in direct interaction with tissue cells, the cell viability test was performed as a first criterion. Pre-osteoblastic cells (MC3T3-E1) were cultured on PCL and the composite PCLNT-4 for periods of up to 7 days, and the cell proliferation result was compared with that of pure PCL. Cells grown on the PCL and PCLNT-4 after 3 days of culturing were stained and the nuclei of viable cells were revealed by
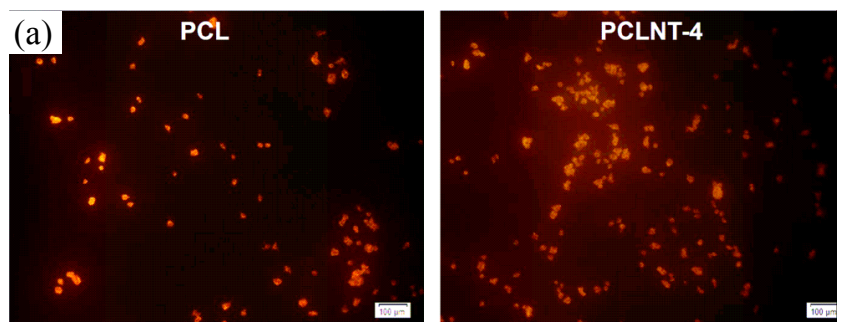

(b)

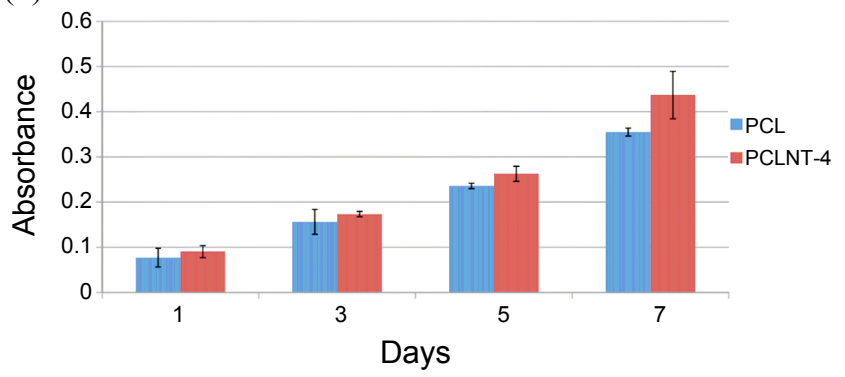

Figure 5. (a) Fluorescence microscopic images of cell growth on PCL and PCLNT-4 for 3 days of culturing and (b) cell proliferation level quantified by MTS method during culturing for up to 7 days. 
fluorescence microscopy (Figure 5a). More viable cells appeared to be present on the PCLNT-4 nanocomposites as compared with those on pure PCL. The cell proliferation level was quantified by MTS method (Figure 5b) during culturing up to 7 days. Cell proliferation was better on the PCLNT-4 nanocomposite than on the pure PCL and the difference was significant at 7 days. At least from the results, the produced nanocomposites are considered to provide favorable substrate condition for tissue cells to adhere, spread and proliferate. Further assessments on cellular or tissue responses to the PCLNT composites in relation with tissue specific applications are currently underway.

\section{Conclusion}

Novel nanocomposites composed of PCL and MWCNTs were developed using the chemically-modified MWCNTs that could be effectively dispersed in non-aqueous solvents. PCL was smoothly dissolved in the nanotubes solution, resulting in the formation of highly clean and homogeneous composite solutions without agglomeration of the nanotubes. The concentration of nanotubes in the nanocomposites was tunable simply by adjusting the used quantity of the MWCNT-Cl solution. In the presence of relatively small amount of MWCNT-Cl (0.25 $0.5 \mathrm{wt} \%$ ), the strength increased by about $34.4 \%$. According to cell viability test, the nanocomposites are considered to provide favorable substrate condition for tissue cells to adhere, spread and proliferate. The approach to using the chemically-modified MWCNTs is considered to open a new door to the class of CNTadded polymer nanocomposites, including those for biomedical applications.
Acknowledgments. This work was supported by a grant from the Healthcare technology R\&D Project, Ministry for Health, Welfare \& Family Affairs (A084491).

\section{References}

1. Tans, S. J.; Devoret, M. H.; Dai, H.; Thess, A.; Smalley, R. E.; Geerligs, L. J.; Dekker, C. Nature 1997, 386, 474.

2. Zhang, W. D.; Phang, I. Y.; Liu, T. X. AdV. Mater. 2006, 18, 73.

3. Moon, S. I.; Jin, F.; Lee, C. J.; Tsutsumi, S.; Hyon, S. H. Macromol. Symp. 2005, 224, 287.

4. Liu, J.; Rinzler, A. G.; Dai, H.; Hafner, J. H.; Bradley, R. K.; Boul, P. J.; Lu, A.; Iverson, T.; Shelimov, K.; Huffman, C. B.; Rodriguez-Macias, F.; Shon, Y. S.; Lee, T. R.; Colbert, D. T.; Smalley, R. E. Science 1998, 280, 1253.

5. Wang, Y.; Iqbal, Z.; Mitra, S. J. Am. Chem. Soc. 2006, 128, 95.

6. Chiu, W.-M.; Chang, Y.-A.; Kuo, H.-Y.; Lin, M.-H.; Wen, H.-C. J. Appl. Poly. Sci. 2008, 108, 3024.

7. Kumar, N. A.; Ganapathy, H. S.; Kim, J.-S.; Jeong, Y.-S.; Jeong, Y.-T. Eur. Polym. J. 2008, 44, 579.

8. Zhao, Y.; Qiu, Z.; Yang, W. J. Phys. Chem. B 2008, 112, 16461.

9. Chen, G.-X.; Shimizu, H. Polymer 2008, 49, 943.

10. Liu, T.; Phang, I. Y.; Shen, L.; Chow, S. Y.; Zhang, W.-D. Macromolecules 2004, 37, 7214.

11. Gao, J.; Zhao, B.; Itkis, M. E.; Bekyarova, E.; Hu, H.; Kranak, V.; Yu, A.; Haddon, R. C. J. Am. Chem. Soc. 2006, 128, 7492.

12. Zeng, H.; Gao, C.; Yan, D. Adv. Funct. Mater. 2006, 16, 812.

13. Van Natta, F. J.; Hill, J. W.; Carruthers, W. H. J. Am. Chem. Soc. 1934, 56, 455 .

14. Sinha, V. R.; Bansal, K.; Kaushik, R.; Kumria, R.; Trehan, A. Int. J. Pharm. 2004, 278, 1.

15. Choi, D. S.; Kim, J. H.; Shin, U. S.; Deshmukh, R. R.; Song, C. E. Chem. Commun. 2007, 3482. 